\title{
Existence of Solutions for Boundary Value Problem of a Caputo Fractional Difference Equation
}

\author{
Zhiping Liu, ${ }^{1}$ Shugui Kang, ${ }^{2}$ Huiqin Chen, ${ }^{2}$ Jianmin Guo, ${ }^{2}$ Yaqiong Cui, ${ }^{2}$ and Caixia Guo \\ ${ }^{1}$ Department of Mathematics, North University of China, Shanxi, Taiyuan 030051, China \\ ${ }^{2}$ School of Mathematics and Computer Sciences, Shanxi Datong University, Datong, Shanxi 037009, China \\ Correspondence should be addressed to Shugui Kang; dtkangshugui@126.com
}

Received 6 June 2015; Revised 22 July 2015; Accepted 26 July 2015

Academic Editor: Chris Goodrich

Copyright (C) 2015 Zhiping Liu et al. This is an open access article distributed under the Creative Commons Attribution License, which permits unrestricted use, distribution, and reproduction in any medium, provided the original work is properly cited.

We investigate the existence of solutions for a Caputo fractional difference equation boundary value problem. We use Schauder fixed point theorem to deduce the existence of solutions. The proofs are based upon the theory of discrete fractional calculus. We also provide some examples to illustrate our main results.

\section{Introduction}

The theory of fractional difference equations and their applications have been receiving intensive attention. In the last ten years, new research achievements kept emerging (see [1-16] and the references therein). In [1-3], the authors introduced fractional sum and difference operators, studied their behavior, and developed a complete theory governing their compositions. Abdeljawad [3] considered the initial value problem to Caputo fractional difference equation. The authors explored BVP of fractional difference equation, and they deduced the existence of one or more positive solutions in [4-7]. Abdeljawad and Baleanu [8] introduced the fractional differences and integration by parts. In [9] the authors studied the stability of discrete nonautonomous systems within the frame of the Caputo fractional difference by using Lyapunov's direct method. They discussed the conditions for uniform stability, uniform asymptotic stability, and uniform global stability.

In $[10,11]$, the authors studied multiple solutions to fractional difference boundary value problems by means of Krasnosel'skii theorem and Schauder fixed point theorem. They obtained sufficient conditions of the existence of two positive solutions for the boundary value problem of fractional difference equations depending on parameters in [12].
Chen et al. [13] presented the existence of at least one positive solution for Caputo fractional boundary value problems. In [14], Kang et al. discussed existence of positive solutions for a system of Caputo fractional difference equations depending on parameters on the basis of [13].

Recently, Wu and Baleanu introduced some applications of the Caputo fractional difference to discrete chaotic maps in $[15,16]$. Thus, the fractional difference equation has recently attracted increasing attention from a growing number of researchers.

Following this trend, we investigate the following boundary value problem of fractional difference equation (FBVP):

$$
\begin{aligned}
& \Delta_{C}^{v} y(t)=-f(t+v-1, y(t+v-1)), \\
& y(v-3)=\Delta y(b+v)=\Delta^{2} y(v-3)=0,
\end{aligned}
$$

where $t \in\{0,1, \ldots, b+1\}:=[0, b+1]_{\mathbb{N}_{0}}, b \geqslant 5$, is an integer. $f:[\nu-2, b+v]_{\mathbb{N}_{\nu-2}} \times \mathbb{R} \rightarrow \mathbb{R}$ is continuous and $f$ is not identically zero, $2<v \leqslant 3$, and $\Delta_{C}^{v} y(t)$ is the standard Caputo difference.

The reason for studying (1) is that, in [13], Chen et al. studied similar FBVP (1) by using the cone fixed point theorem. In this note, we consider existence of solutions to 
FBVP (1) by using Schauder theorem. Here, the nonnegative function $f$ is not necessary, but $f$ is nonnegative in [13]. We extend the domain of $f$.

This paper is organized as follows. Section 1 introduces the developing of fractional difference equations in a simple way. We present some necessary definitions and lemmas in Section 2. In Section 3, we prove existence of solution to FBVP (1). Finally, we provide some examples to illustrate our main results.

\section{Preliminaries}

We first introduce some theory about fractional sums and differences. The definitions and some basic results about fractional sums and differences can be seen in [1-7], so we omit their proof.

Definition 1 (see [3]). For any $t$ and $\nu$, one defines

$$
t^{\underline{v}}=\frac{\Gamma(t+1)}{\Gamma(t+1-v)}
$$

for which the right-hand side is defined. One appeals to the convention that if $t+1-v$ is a pole of the Gamma function and $t+1$ is not a pole, then $t^{\underline{v}}=0$.

Definition 2 (see [3]). The $v$ th fractional sum of a function $f$ is

$$
\Delta^{-\nu} f(t)=\frac{1}{\Gamma(\nu)} \sum_{s=a}^{t-v}(t-s-1)^{\frac{\nu-1}{}} f(s)
$$

for $\nu>0$ and $t \in\{a+v, a+v+1, \ldots\}:=\mathbb{N}_{a+v}$. One also defines the $v$ th Caputo fractional difference for $v>0$ by

$$
\begin{aligned}
\Delta_{C}^{\nu} f(t) & =\Delta^{-(n-v)} \Delta^{n} f(t) \\
& =\frac{1}{\Gamma(n-v)} \sum_{s=a}^{t-(n-v)}(t-s-1)^{\frac{n-\nu-1}{2}} \Delta_{a}^{n} f(s),
\end{aligned}
$$

where $n-1<v \leqslant n$.

Lemma 3 (see [3]). Assume that $v>0$ and $f$ is defined on domains $\mathbb{N}_{a}$, then

$$
\Delta_{a+(n-v)}^{-v} \Delta_{C}^{v} f(t)=f(t)-\sum_{k=0}^{n-1} c_{k}(t-a)^{\underline{k}},
$$

where $c_{k} \in \mathbb{R}, k=0,1, \ldots, n-1$, and $n-1<v \leqslant n$.

Lemma 4 (see $[13])$. Let $2<v \leqslant 3$ and $g:[\nu-2, b+v]_{\mathbb{N}_{\nu-2}} \rightarrow$ $\mathbb{R}$ be given. Then the solution of FBVP,

$$
\begin{aligned}
& \Delta_{C}^{v} y(t)=-g(t+v-1), \\
& y(v-3)=\Delta y(b+v)=\Delta^{2} y(v-3)=0,
\end{aligned}
$$

is given by

$$
y(t)=\sum_{s=0}^{b+1} G(t, s) g(s+v-1),
$$

where Green's function $G:[\nu-2, b+v]_{\mathbb{N}_{\nu-2}} \times[0, b+1]_{\mathbb{N}_{0}} \rightarrow \mathbb{R}$ is defined by

$$
G(t, s)=\frac{1}{\Gamma(\nu)} \begin{cases}(\nu-1)(t-v+3)(b+v-s-1)^{\frac{\nu-2}{}-(t-s-1)^{\frac{\nu-1}{2},}} & 0 \leqslant s<t-v+1 \leqslant b+1, \\ (\nu-1)(t-v+3)(b+v-s-1)^{\frac{\nu-2}{},} & 0 \leqslant t-v+1 \leqslant s \leqslant b+1 .\end{cases}
$$

Remark 5. Notice that $G(\nu-3, s)=0, G(t, b+2)=0$. $G$ could be extended to $[\nu-3, b+\nu]_{\mathbb{N}_{\nu-3}} \times[0, b+2]_{\mathbb{N}_{0}}$, so we only discuss $(t, s) \in[v-2, b+v]_{\mathbb{N}_{\nu-2}} \times[0, b+1]_{\mathbb{N}_{0}}$.

Lemma 6 (see [13]). The Green function $G$ has the following properties:

(i) $G(t, s)>0,(t, s) \in[\nu-2, b+v]_{\mathbb{N}_{\nu-2}} \times[0, b+1]_{\mathbb{N}_{0}}$.

(ii) $\max _{t \in[v-2, b+v]_{\mathbb{N}-2}} G(t, s)=G(b+v, s), s \in[0, b+1]_{\mathbb{N}_{0}}$.

Let

$$
\begin{aligned}
\mathscr{K} & =\left\{y \mid y:[\nu-3, v+b]_{\mathbb{N}_{v-3}} \longrightarrow \mathbb{R}, y(\nu-3)\right. \\
& \left.=\Delta y(\nu+b)=\Delta^{2} y(\nu-3)=0\right\} .
\end{aligned}
$$

It is clear that $\mathscr{K}$ is a Banach space with the norm $\|y\|=$ $\max _{t \in[v-2, v+b]_{\mathbb{N}_{\nu-2}}}|y(t)|$. Now consider the operator $T$ defined by

$$
T(y)(t)=\sum_{s=0}^{b+1} G(t, s) f(s+v-1, y(s+v-1))
$$

where $t \in[\nu-2, b+v]_{\mathbb{N}_{\nu-2}}, f:[\nu-2, b+v]_{\mathbb{N}_{\nu-2}} \times \mathbb{R} \rightarrow \mathbb{R}$ is continuous, and $f$ is not identically zero. It is easy to see that $y=y(t)$ is a solution of FBVP (1) if and only if $y=y(t)$ is a fixed point of $T$.

Lemma 7 (see [17] (Schauder fixed point theorem)). Suppose that $\mathscr{K}$ is a Banach space. Let $\mathscr{B}$ be a bounded closed convex set of $\mathscr{K}$, and let $T: \mathscr{B} \rightarrow \mathscr{B}$ be a complete continuous operator. Then $T$ has at least one fixed point in $\mathscr{B}$. 


\section{Main Results}

In this section, we give the main result of this paper. We will prove this result by using Schauder fixed point theorem and provide some examples to illustrate our main results.

For the sake of convenience, we write out the conditions as follows:

$\left(H_{1}\right)$ There exists a nonnegative function $a \in C([\nu-2, b+$ $v]_{\mathbb{N}_{\nu-2}}$ ) and a constant $c$ such that $|f(t, y)| \leqslant a(t)+$ $c|y|^{\rho}$, where $c \geqslant 0,0<\rho<1$.

$\left(H_{2}\right)|f(t, y)| \leqslant c|y|^{\rho}$, where $c>0, \rho>1$.

Theorem 8. Let $f:[v-2, b+v]_{\mathbb{N}_{v-2}} \times \mathbb{R} \rightarrow \mathbb{R}$ be $a$ continuous function. Suppose that one of conditions $\left(H_{1}\right)$ and $\left(\mathrm{H}_{2}\right)$ is satisfied. Then FBVP (1) has at least one solution.

Proof. First, suppose that condition $\left(H_{1}\right)$ is satisfied. Let

$\mathscr{B}$

$$
=\left\{y(t) \mid y(t) \in \mathscr{K},\|y\| \leqslant R, t \in[\nu-2, b+\nu]_{\mathbb{N}_{v-2}}\right\},
$$

where

$$
\begin{aligned}
& R \geqslant \max \left\{2 \sum_{s=0}^{b+1} G(b+v, s)|a(s+v-1)|,\right. \\
& {\left.\left[2 c \frac{\Gamma(b+v+1)}{\Gamma(b+1) \Gamma(\nu)}\left(\frac{1}{v}+\frac{b+3}{b+1}\right)\right]^{1 /(1-\rho)}\right\} . }
\end{aligned}
$$

Obviously $\mathscr{B}$ is the ball in the Banach space.

Now we prove that $T: \mathscr{B} \rightarrow \mathscr{B}$. For any $y \in \mathscr{B}$, then

$$
\begin{aligned}
& |(T y)(t)| \\
& \quad \leqslant \sum_{s=0}^{b+1}\left|G(t, s)\left[a(s+v-1)+c|y(s+v-1)|^{\rho}\right]\right| \\
& \quad \leqslant \sum_{s=0}^{b+1}|G(t, s) a(s+v-1)|+\sum_{s=0}^{b+1} c\|y\|^{\rho} \cdot|G(t, s)| \\
& \quad \leqslant \sum_{s=0}^{b+1} G(b+v, s)|a(s+v-1)|+c R^{\rho}\left(\sum_{s=0}^{t-v}|G(t, s)|\right. \\
& \left.+\sum_{s=t-v+1}^{b+1}|G(t, s)|\right) \leqslant \sum_{s=0}^{b+1} G(b+v, s)|a(s+v-1)| \\
& +\frac{c R^{\rho}}{\Gamma(\nu)} \sum_{s=0}^{t-v}(t-s-1) \frac{v-1}{c}+\frac{c R^{\rho}}{\Gamma(\nu)} \sum_{s=0}^{b+1}(\nu-1)
\end{aligned}
$$

$$
\begin{aligned}
& \cdot(t-v+3)(b+v-s-1) \frac{\nu-2}{b+1} \leqslant(b+v, s) \\
& \cdot|a(s+\nu-1)|+c R^{\rho} \max _{t \in[\nu-2, \nu+b]_{\mathbb{N}-2}}\left(\frac{1}{\Gamma(\nu)}\right. \\
& \left.\cdot \sum_{s=0}^{t-\nu}(t-s-1)^{\frac{\nu-1}{}}\right)+c R^{\rho} \max _{t \in[\nu-2, \nu+b]_{\mathbb{N}-2}}\left(\frac{t-\nu+3}{\Gamma(\nu-1)}\right. \\
& \left.\cdot \sum_{s=0}^{b+1}(b+v-s-1)^{\frac{\nu-2}{}}\right) \text {. }
\end{aligned}
$$

As

$$
\begin{aligned}
& \frac{1}{\Gamma(\nu)} \sum_{s=0}^{t-\nu}(t-s-1)^{\frac{\nu-1}{}}=\frac{1}{\Gamma(\nu)}\left[-\frac{1}{\nu}(t-s)^{\underline{\nu}}\right]_{s=0}^{t-\nu+1} \\
& =\frac{t^{\underline{\nu}}}{\Gamma(\nu+1)} \leq \frac{\Gamma(b+\nu+1)}{\Gamma(b+1) \Gamma(\nu+1)}, \\
& \frac{t-v+3}{\Gamma(\nu-1)} \sum_{s=0}^{b+1}(b+v-s-1)^{\frac{v-2}{2}} \\
& \leqslant \frac{b+3}{\Gamma(\nu-1)}\left[-\frac{1}{v-1}(b+v-s)^{\frac{\nu-1}{b+2}}\right]_{s=0}^{b} \\
& =\frac{(b+3) \Gamma(b+v+1)}{\Gamma(b+2) \Gamma(\nu)},
\end{aligned}
$$

it follows that

$$
\begin{aligned}
|(T y)(t)| \leqslant & \sum_{s=0}^{b+1} G(b+v, s)|a(s+v-1)| \\
& +c R^{\rho} \frac{\Gamma(b+v+1)}{\Gamma(b+1) \Gamma(v+1)} \\
& +c R^{\rho} \frac{(b+3) \Gamma(b+v+1)}{\Gamma(b+2) \Gamma(v)} \\
= & \sum_{s=0}^{b+1} G(b+v, s)|a(s+v-1)| \\
& +c R^{\rho} \frac{\Gamma(b+v+1)}{\Gamma(b+1) \Gamma(v)}\left[\frac{1}{v}+\frac{b+3}{b+1}\right] \\
\leqslant & \frac{R}{2}+\frac{R}{2}=R .
\end{aligned}
$$

Hence, $\|T y\| \leqslant R$. Namely, $T: \mathscr{B} \rightarrow \mathscr{B}$.

Second, let condition $\left(\mathrm{H}_{2}\right)$ be valid. Choose

$$
0<R \leqslant\left[\frac{\Gamma(\nu) \Gamma(b+1)}{c \Gamma(b+v+1)}\left(\frac{1}{v}+\frac{b+3}{b+1}\right)^{-1}\right]^{1 /(\rho-1)} .
$$

Repeating the course of the above, we obtain $|T(y)| \leqslant$ $R$. Consequently, we get $T: \mathscr{B} \rightarrow \mathscr{B}$. By means of the 
continuity of $G$ and $f$, it is easy to see that operator $T$ is continuous. Next, we show that $T$ is a completely continuous operator. For this, we take

$$
M=\max _{t \in[\nu-2, b+\nu]_{\mathbb{N}-2}}|f(t, y(t))|
$$
then

For any $y \in \mathscr{B}$, let $t, \tau \in[\nu-2, b+\nu]_{\mathbb{N}_{v-2}}$ such that $t<\tau$;

$$
\begin{aligned}
& |(T y)(t)-(T y)(\tau)|=\sum_{s=0}^{b+1} \mid(G(t, s)-G(\tau, s)) \\
& \cdot f(s+v-1, y(s+v-1)) \mid \\
& \leqslant M\left[\sum_{s=0}^{t-\gamma}|G(t, s)-G(\tau, s)|\right. \\
& +\sum_{s=t-\nu+1}^{\tau-v}|G(t, s)-G(\tau, s)| \\
& \left.+\sum_{s=\tau-\nu+1}^{b+1}|G(t, s)-G(\tau, s)|\right]=\frac{M}{\Gamma(\nu)} \sum_{s=0}^{t-v} \mid(\nu-1) \\
& \cdot(t-v+3)(b+v-s-1)^{\frac{\nu-2}{2}}-(t-s-1)^{\frac{\nu-1}{}} \\
& -(\nu-1)(\tau-v+3)(b+v-s-1)^{\frac{\nu-2}{2}} \\
& +(\tau-s-1)^{\frac{\nu-1}{}}\left|+\frac{M}{\Gamma(\nu)} \sum_{s=t-\nu+1}^{\tau-\nu}\right|(\nu-1)(t-\nu+3) \\
& \cdot(b+v-s-1)^{\frac{\nu-2}{2}}-(\nu-1)(\tau-\nu+3) \\
& \cdot(b+v-s-1)^{\frac{\nu-2}{2}}\left|+\frac{M}{\Gamma(\nu)} \sum_{s=\tau-\nu+1}^{b+1}\right|(\nu-1) \\
& \cdot(t-v+3)(b+v-s-1) \frac{v-2}{2}-(\nu-1)(\tau-v+3) \\
& \cdot(b+v-s-1)^{\frac{\nu-2}{}}\left|\leqslant \frac{M}{\Gamma(\nu)} \sum_{s=0}^{t-v}\right|(\nu-1) \\
& \cdot(b+v-s-1)^{\frac{\nu-2}{2}}(t-\tau)+(\tau-s-1)^{\frac{\nu-1}{2}} \\
& -(t-s-1)^{\frac{\nu-1}{}}\left|+\frac{M}{\Gamma(\nu)} \sum_{s=t-v+1}^{\tau-v}\right|(\nu-1) \\
& \cdot(b+\nu-s-1)^{\frac{\nu-2}{2}}(t-\tau)+(\tau-s-1)^{\frac{\nu-1}{}} \mid+\frac{M}{\Gamma(\nu)} \\
& \cdot \sum_{s=\tau-\nu+1}^{b+1}\left|(\nu-1)(b+\nu-s-1)^{\frac{\nu-2}{2}}(t-\tau)\right| \\
& =\frac{M}{\Gamma(\nu)}\left[\sum_{s=0}^{b+1}(\nu-1)(b+\nu-s-1)^{\frac{\nu-2}{2}}|t-\tau|\right. \\
& \left.+\sum_{s=0}^{\tau-\gamma}(\tau-s-1)^{\frac{\nu-1}{}}-\sum_{s=0}^{t-v}(t-s-1)^{\frac{\nu-1}{}}\right]
\end{aligned}
$$

$$
\begin{aligned}
& =\frac{M}{\Gamma(\nu)}\left[\left[-(b+\nu-s)^{\frac{\nu-1}{}}\right]_{s=0}^{b+2}|t-\tau|\right. \\
& \left.+\left[-\frac{1}{\nu}(\tau-s)^{\underline{\nu}}\right]_{s=0}^{\tau-\nu+1}-\left[-\frac{1}{\nu}(t-s)^{\underline{\nu}}\right]_{s=0}^{t-\nu+1}\right] \\
& =\frac{M}{\Gamma(\nu)}\left[\left[-(\nu-2)^{\frac{\nu-1}{}}+(b+\nu)^{\frac{\nu-1}{}}\right]|t-\tau|+\frac{1}{\nu}\left(\tau^{\underline{\nu}}\right.\right. \\
& \left.\left.-t^{\underline{\nu}}\right)\right]=\frac{M}{\Gamma(\nu)}\left[(b+\nu)^{\frac{\nu-1}{}}|t-\tau|+\frac{1}{\nu}\left(\tau^{\underline{\nu}}-t^{\underline{\nu}}\right)\right] .
\end{aligned}
$$

Since functions $t^{\underline{v}}, t$ are uniformly continuous on interval $[\nu-2, b+\nu]_{\mathbb{N}_{v-2}}$, we conclude that $T(\mathscr{B})$ is an equicontinuous set. Obviously, it is uniformly bounded since $T(\mathscr{B}) \subset \mathscr{B}$. Thus, we know $T$ is completely continuous.

Consequently, it follows at once by Schauder fixed point theorem that $T$ has a fixed point $y$; namely, $y$ is a solution of (1). The theorem is proved.

Remark 9. In this paper, $f$ is only continuous function, without nonnegative assumptions on function $f$.

Remark 10. If $\rho=1$ in $\left(H_{1}\right)$, we need condition $c(\Gamma(\nu+b+$ $1) / \Gamma(b+1) \Gamma(\nu))[1 / \nu+(b+3) /(b+1)] \leqslant 1 / 2$. At this moment, choose

$$
R \geqslant 2 \sum_{s=0}^{b+1} G(s+v-1, s)|a(s+v-1)| .
$$

If $\rho=1 \mathrm{in}\left(H_{2}\right)$, we only need condition $c(\Gamma(\nu+b+1) / \Gamma(b+$ $1) \Gamma(\nu))[1 / v+(b+3) /(b+1)] \leqslant 1$. Then the conclusion of Theorem 8 remains true.

Example 11. Consider the following Caputo fractional difference boundary value problem:

$$
\begin{aligned}
& \Delta_{C}^{9 / 4} y(t)=-\sin \pi\left(t+\frac{5}{4}\right)\left(1+\frac{y^{1 / 2}(t+5 / 4)}{y^{2}(t+5 / 4)+50}\right), \\
& y\left(-\frac{3}{4}\right)=\Delta y\left(\frac{45}{4}\right)=\Delta^{2} y\left(-\frac{3}{4}\right)=0,
\end{aligned}
$$

where $v=9 / 4, b=9, f(t, y)=\sin \pi t \cdot\left(1+y^{1 / 2} /\left(y^{2}+50\right)\right)$. Since

$$
|f(t, y)| \leqslant 1+\frac{1}{50} y^{1 / 2}
$$

then $a(t)=1, c=1 / 50$, and $\rho=1 / 2$. At this moment, we take $R=270.918$. The condition of $\left(H_{1}\right)$ in Theorem 8 is satisfied. Applying Theorem 8, FBVP (20) has at least one solution $y$, and $|y| \leqslant R$.

Example 12. Consider the following Caputo fractional difference boundary value problem:

$$
\begin{gathered}
\Delta_{C}^{5 / 2} y(t)=-\frac{2 \sin \pi(t+3 / 2)}{2 t+323} y^{2}\left(t+\frac{3}{2}\right), \\
y\left(-\frac{1}{2}\right)=\Delta y\left(\frac{23}{2}\right)=\Delta^{2} y\left(-\frac{1}{2}\right)=0,
\end{gathered}
$$


where $t \in[0,10]_{\mathbb{N}_{0}}$ and $v=5 / 2, b=9$, and $f(t, y)=$ $(\sin \pi t /(t+160)) y^{2}$. As

$$
|f(t, y)| \leqslant \frac{1}{160} y^{2}
$$

then $a(t)=0, c=1 / 160$, and $\rho=2>1$. At this moment, we take $R=0.3525$. The condition of $\left(\mathrm{H}_{2}\right)$ in Theorem 8 is satisfied. Applying Theorem 8, FBVP (22) has at least one solution $y$, and $|y| \leqslant R$.

\section{Conflict of Interests}

The authors declare that there is no conflict of interests regarding the publication of this paper.

\section{Acknowledgments}

The authors are very grateful to the referee for her/his valuable suggestions. This paper is supported by the National Natural Science Foundation of China (Grant no. 11271235) and Shanxi Datong University Institute (2013K5).

\section{References}

[1] M. Holm, "Sum and difference compositions in discrete fractional calculus," Cubo, vol. 13, no. 3, pp. 153-184, 2011.

[2] F. M. Atici and P. W. Eloe, "Discrete fractional calculus with the nabla operator," Electronic Journal of Qualitative Theory of Differential Equations, vol. 3, pp. 1-12, 2009.

[3] T. Abdeljawad, "On Riemann and Caputo fractional differences," Computers \& Mathematics with Applications, vol. 62, no. 3, pp. 1602-1611, 2011.

[4] C. S. Goodrich, "Some new existence results for fractional difference equations," International Journal of Dynamical Systems and Differential Equations, vol. 3, no. 1-2, pp. 145-162, 2011.

[5] C. S. Goodrich, "On positive solutions to nonlocal fractional and integer-order difference equations," Applicable Analysis and Discrete Mathematics, vol. 5, no. 1, pp. 122-132, 2011.

[6] C. S. Goodrich, "Existence of a positive solution to a system of discrete fractional boundary value problems," Applied Mathematics and Computation, vol. 217, no. 9, pp. 4740-4753, 2011.

[7] C. S. Goodrich, "On a discrete fractional three-point boundary value problem," Journal of Difference Equations and Applications, vol. 18, no. 3, pp. 397-415, 2012.

[8] T. Abdeljawad and D. Baleanu, "Fractional differences and integration by parts," Journal of Computational Analysis and Applications, vol. 13, no. 3, pp. 574-582, 2011.

[9] F. Jarad, T. Abdeljawad, D. Baleanu et al., "On the stability of some discrete fractional nonautonomous systems," Abstract and Applied Analysis, vol. 2012, Article ID 476581, 9 pages, 2012.

[10] H. Q. Chen, Y. Q. Cui, and X. L. Zhao, "Multiple solutions to fractional difference boundary value problems," Abstract and Applied Analysis, vol. 2014, Article ID 879380, 6 pages, 2014.

[11] S. G. Kang, Y. Li, and H. Q. Chen, "Positive solutions to boundary value problems of fractional difference equation with nonlocal conditions," Advances in Difference Equations, vol. 2014, article 7, 12 pages, 2014.

[12] S. Kang, X. Zhao, and H. Chen, "Positive solutions for boundary value problems of fractional difference equations depending on parameters," Advances in Difference Equations, vol. 2013, article 376, 2013.
[13] H. Q. Chen, Z. Jin, and S. G. Kang, "Existence of positive solutions for Caputo fractional difference equation," Advances in Difference Equations, vol. 44, pp. 1-12, 2015.

[14] S. G. Kang, H. Q. Chen, and J. M. Guo, "Existence of positive solutions for a system of Caputo fractional difference equations depending on parameters," Advances in Difference Equations, vol. 2015, article 138, 2015.

[15] G.-C. Wu and D. Baleanu, "Discrete fractional logistic map and its chaos," Nonlinear Dynamics, vol. 75, no. 1-2, pp. 283-287, 2014.

[16] G.-C. Wu and D. Baleanu, "Chaos synchronization of the discrete fractional logistic map," Signal Processing, vol. 102, pp. 96-99, 2014.

[17] R. P. Agarwal, M. Meehan, and D. O’Regan, Fixed Point Theory and Applications, Cambridge University Press, Cambridge, UK, 2001. 


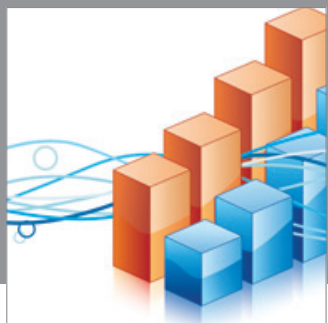

Advances in

Operations Research

mansans

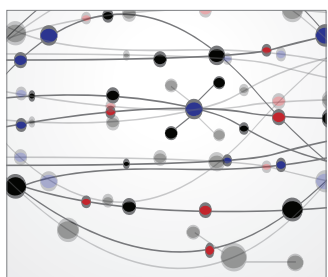

The Scientific World Journal
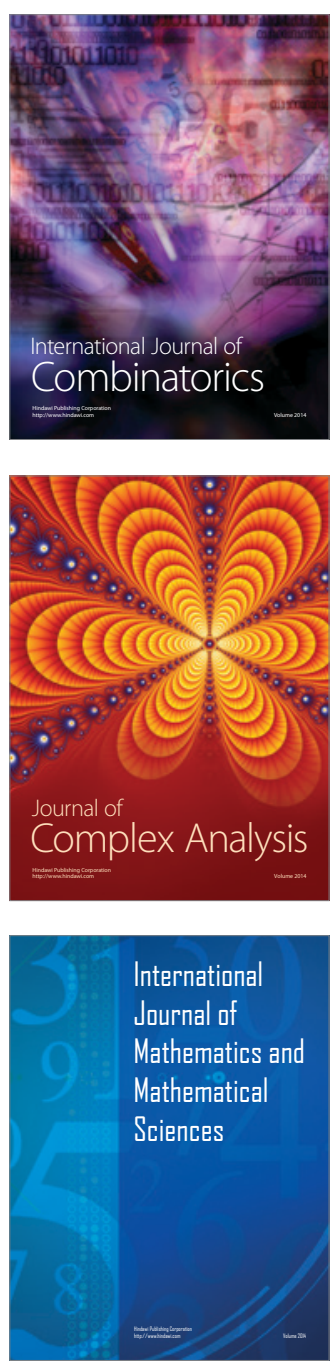
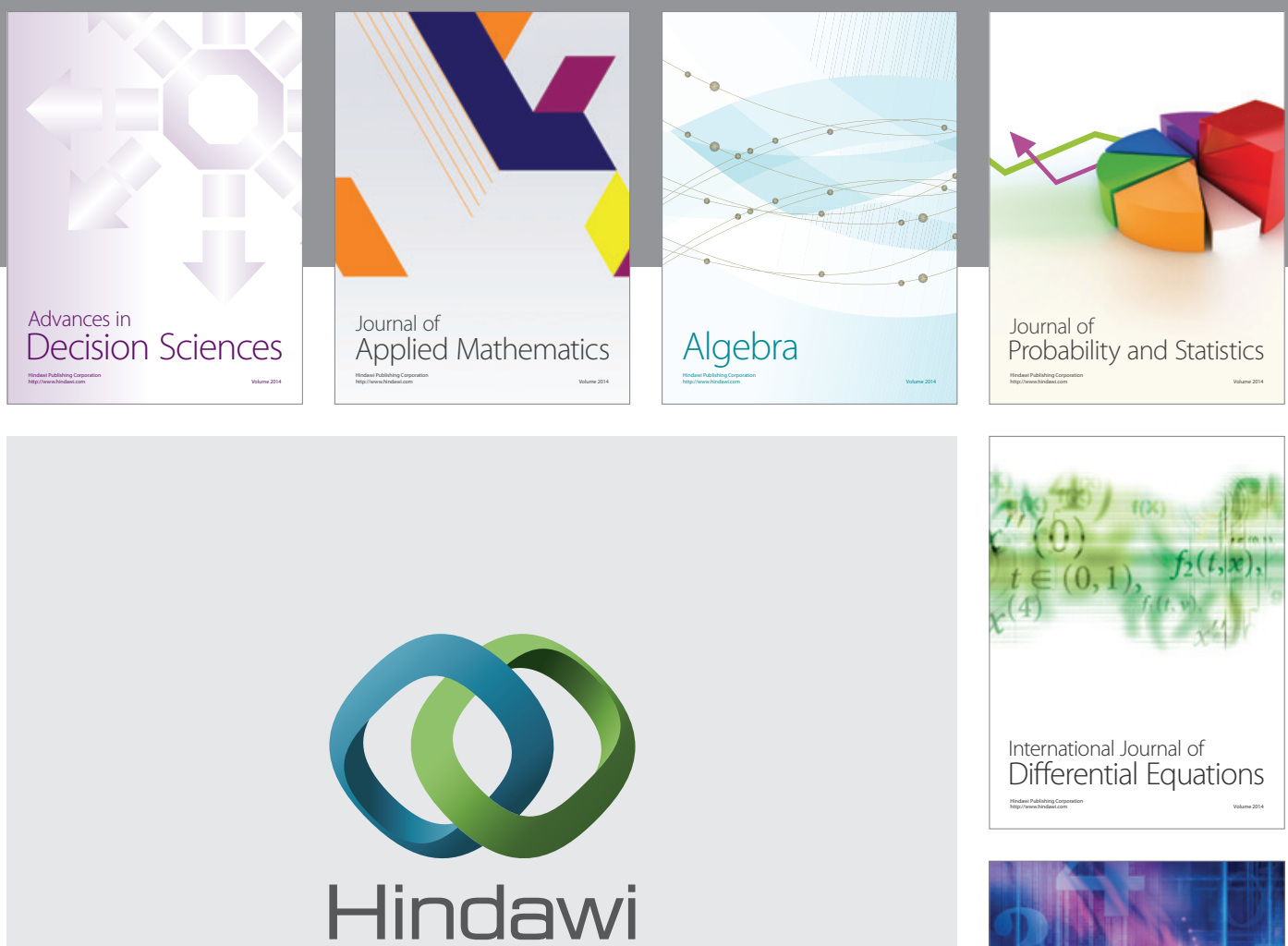

Submit your manuscripts at http://www.hindawi.com
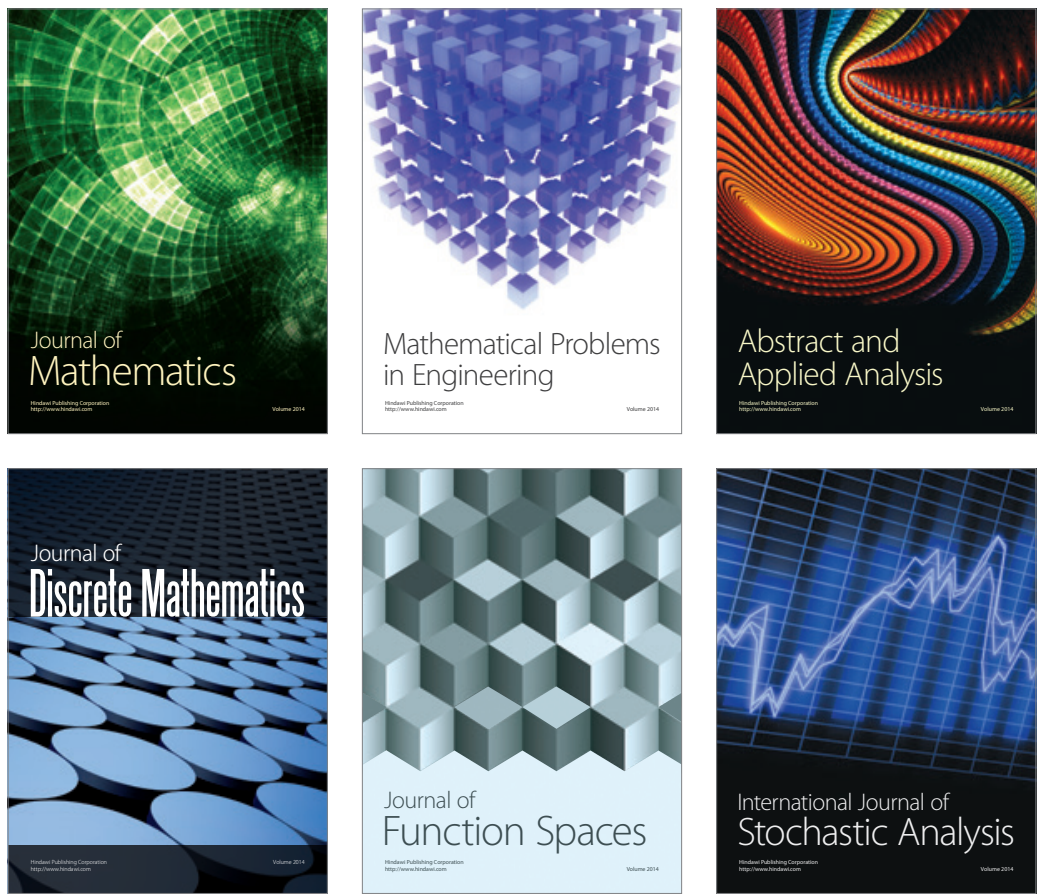

Journal of

Function Spaces

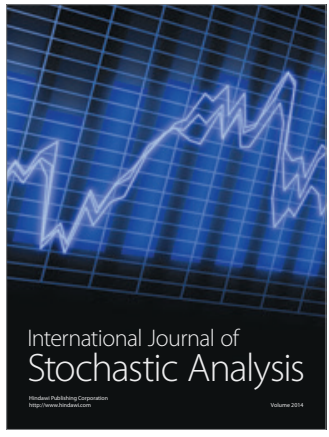

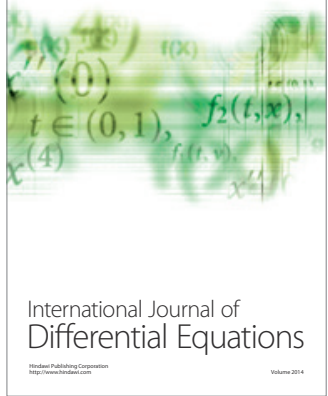
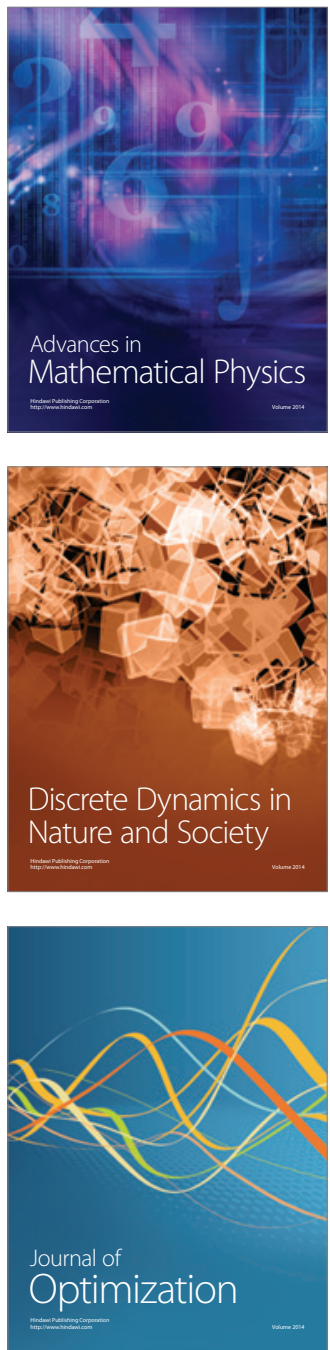